\title{
Methods of Measuring Musical Tension: Commentary on Teo (2020)
}

\author{
CAITLYN TREVOR[1] \\ Department of Psychology, University of Zurich
}

\begin{abstract}
This commentary discusses the motivation, methods, and results of Teo's (2020) article on using Aggregate Voice-Leading (AVL) motion to predict perceived harmonic tension within post-tonal, neoclassical repertoire. The successes of the study as well as possible improvements are furthermore discussed. The final section contemplates some of Teo's results in light of BaileyShea and Monahan's (2018) embodied theory of analyzing and describing musical energetics such as tension. This embodied theory does not rely on tonality to operate and is therefore applicable to many genres of music. It could be an especially useful theory for analyzing genres of music for which past models of harmonic tension are insufficient, such as post-tonal or electroacoustic music.
\end{abstract}

Submitted 2020 May 8; accepted 2020 May 9.

Published 2020 October 22; https://doi.org/10.18061/emr.v15i1-2.7660

KEYWORDS: musical tension, post-tonal, voice-leading, embodiment

WHILE music theory has long pursued the question of how musical tension is perceived within tonal and atonal music, little has been discussed regarding the intersection of those two categories: post-tonal music. Motivated by the need to fill this knowledge gap, in her paper "Measuring Harmonic Tension in Post-Tonal Repertoire", Teo (2020) tested whether theoretically derived harmonic tension could be used to predict perceived tension in three neoclassical pieces by Hindemith, Ravel, and Stravinsky. To theoretically quantify harmonic tension, Teo calculated the total sum of all voice-leading movement between chords (specifically pitch collections, PCns), a method derived from aspects of Schenkerian, neo-Riemannian, and pitch-class set theories. Teo refers to this calculation as the Aggregated VoiceLeading (AVL) movement. To measure the perceived tension of movements between PCns, Teo had 63 participants listen to excerpts from the three pieces of interest and then rate the tension of smaller two-chord segments from within those larger excerpts on a scale from 1 to 5. Additionally, Teo measured other characteristics of the PCns, including tonal stability and roughness using the MIR Toolbox (Lartillot, Toiviainen, \& Eerola, 2008) and mean pitch, median range, and number of notes in a PCn (chord size) using the MIDI toolbox (Eerola \& Toiviainen, 2004). Teo's main $a$ priori hypothesis was that AVL values would positively correlate with mean tension ratings (MTRs) - i.e. more voiceleading motion would be perceived as more tense. Additionally, Teo predicted that the MTRs would be biased by the harmonic context that the PCns appeared in and by the overall amount of voice-leading motion present in each excerpt. Teo further predicted that the MTRs would also be related to other preconceived notions of tension and release like consonance/dissonance or register. However, Teo did not make any explicit claims about the impact of these biases or of the other preconceived notions on the results. Ultimately, the results were inconsistent with Teo's main hypothesis in that the AVL values were not correlated with the MTRs. Upon further analysis, other factors appeared to be more powerful predictors of MTRs, especially chord size and tonal stability.[2] Other notable predictors of perceived tension appeared to be register, roughness, and the arrangement of the chords (i.e., root position versus first inversion).

\section{HIGHLIGHTS AND SUGGESTIONS}

Teo's seamless integration of methods and concepts from empirical musicology with more traditional analysis techniques is exemplary and engaging, bridging the sometimes yawning gap between music cognition and music theory. In particular, Teo's method of resolving unexpected values in the data by turning to the score is a wonderful demonstration of how such data-driven tools can be illuminating and relevant within a traditional analysis context. Perhaps Teo's work will encourage some who are dubious of computational methods to reconsider their value. 
While Teo's main hypothesis presented what proved to be an overly simplistic model, it was an admirably bold prediction in pursuit of a potentially valuable analysis tool. However, some aspects of how music generally functions could have hinted, perhaps prior to the experiment, to the fact that the model would be too simple. For example, musical complexity and tension often drop at the end of a musical phrase, in both tonal and atonal works (e.g., in the case of an authentic cadence in a tonal piece, cf. Kostka \& Payne, 2008). Such behavior is vividly evident in Teo's data. Large AVL values that matched small MTRs commonly comprised a larger, more dissonant chord moving to a smaller, more consonant chord, such as a unison. This behavior often coincided with the end of a musical idea. Therefore, large AVL values coincided with a resolution or release in music almost as often as they coincided with tenser moments in the music. Furthermore, stasis can sometimes be used to induce tension in music, such as with a suspension, a sustained dominant chord, or a drone tone. Some of these behaviors, particularly a suspension, appeared in Teo's score analysis of unexpected results in the data (e.g., small AVL combined with larger MTR). Given the commonality and frequency of these behaviors across many genres of music, perhaps Teo could have foreseen that measuring AVL would bundle together moments of release or static forms of harmonically driven suspense with those movement-driven moments of tension. Or perhaps Teo predicted that such cases would occur minimally enough to still allow a trend in the data in the predicted direction. The lack of explicit hypotheses regarding other features measured, such as roughness, is a little surprising; especially given the abundant support that Teo's literature review could have offered for such predictions. The absence of explicit hypotheses gives the impression that the study could be partly exploratory. This ambiguity as to whether the study is exploratory or confirmatory can unfortunately limit the impact of the results.

It might have been useful to include a definition of 'roughness' in the main text of the article rather than just having a note referring to another source for a definition. Firstly, some readers may not have encountered the term before, particularly those with a background or expertise in more traditional music theory. Secondly, several definitions and ways of calculating 'roughness' exist in acoustics and music cognition research. Lartillot and Toiviainen (2007), for example, describe roughness as 'sensory dissonance' and calculate it by "adding the beating provoked by each couple of energy peaks in the spectrum" (p. 238). Contrastingly, Arnal, Flinker, Kleinschmidt, Giraud, and Poeppel (2015) measured roughness using the Modulation Power Spectrum (MPS) - a two-dimensional fast Fourier transform of a spectrogram - and described rough sounds as those that "correspond to amplitude modulations ranging from 30 to $150 \mathrm{~Hz}$ [in the MPS] and typically induce unpleasant, rough auditory percepts" (p. 2051). Given the diversity of meanings that 'roughness' can have, it is vital that the term is clearly defined to avoid confusion when interpreting results.

Furthermore, some technical methodological details are unfortunately omitted. For example, aspects regarding how Teo carried out the online portion of her study are missing, such as how the experiment was distributed (e.g., email list-serves, social media, Amazon Turk), what program the experiment interface was created with (e.g., jspsych, Qualtrics), how data quality was assessed and responses validated, and which musical experience survey was used (e.g., GoldMSI, OMSI). Given today's expanding online methods and the growing support for the open science movement, it is useful for such seemingly mundane details to be included. It would also allow a more accurate assessment of the validity of the results and would enable future researchers to potentially replicate the results.

Finally, regarding the analysis section, it would have been helpful to include the full tension rating scale (15) on the x-axis of the AVL and MTR scatterplots rather than just a portion of it. This omission seems like a lost opportunity given that Teo compares the overall MTRs across the three composers. That comparison could have been more effective with a consistent $\mathrm{X}$-axis covering the entire rating scale. Similarly, the data of all three composers could have been overlaid onto the same scatterplot (perhaps in a separate additional graph), or the three graphs could have been put side by side within the same larger figure. Including 0 on both axes (or the full rating scale available in the perceptual experiment) is sometimes preferable and perceived as more honest in data reporting within the scientific community. However, different views exist on this subject (Poldrack, 2020) making this suggestion more a matter of preference.

\section{EMBODIED MUSICAL ENERGETICS}

Relevant to Teo's work, BaileyShea and Monahan (2018) recently proposed a new method for describing musical energetics (i.e., perceptions of movement or force in music such as tension) that is not reliant on tonality. Instead, their concept is representative of the embodied nature of musical energetics. For example, they suggest that music has a generalized gravity, the concept that upward motion in music (e.g., a rising scale) usually gives the impression of requiring more energy than downward motion in music, regardless of where those motions are in relation to a possible tonal center (BaileyShea \& Monahan, 2018). Another concept they describe is that of registral mass variance, the idea that musical events in lower registers may be embodied as heavier or denser than musical events in a higher 
register (BaileyShea \& Monahan, 2018). It is worthwhile to consider how some of the factors Teo found to be important predictors of perceived musical tension relate to these embodied concepts. Teo found that ascending PCns were rated as more tense than descending PCns, and that shifting from larger chords to smaller chords was sometimes rated as less tense than moving from smaller chords to larger chords. Perhaps generalized gravity and registral mass variance can partly account for these perceptions. If an upward motion between chords communicates greater energy expenditure, perhaps it also communicates more tension. Regarding chord size, the number of PCs in the PCn could be embodied in a similar mass variance concept. In that case, moving from a larger chord to a smaller one could be embodied as a letting go of a certain amount of "mass" and possibly a tangential release of tension. While this discussion is speculative, it is apparent that given how BaileyShea and Monahan's ideas on musical energetics are not reliant on tonality, their concepts could be valuable for analyzing pieces without a clear tonal center, such as posttonal works or electroacoustic music, for which past models of harmonic tension are insufficient. Future work in this area might endeavor to find ways of quantifying BaileyShea and Monahan's concepts to use a similar approach to Teo.

\section{ACKNOWLEDGEMENTS}

This article was copyedited by Niels Christian Hansen and layout edited by Kelly Jakubowski.

\section{NOTES}

[1] Correspondence can be addressed to: Dr. Caitlyn Trevor, University of Zurich, Department of Psychology, Binzmuehlestrasse 14, Box 18, 8050-Zurich, Switzerland. Email: caitlyn.trevor@psychologie.uzh.ch

[2] Teo measured tonal stability according to the Krumhansl-Schmuckler key profiles (Krumhansl, 1990).

\section{REFERENCES}

Arnal, L. H., Flinker, A., Kleinschmidt, A., Giraud, A.-L., \& Poeppel, D. (2015). Human screams occupy a privileged niche in the communication soundscape. Current Biology, 25(15), 2051-2056. https://doi.org/10.1016/j.cub.2015.06.043

BaileyShea, M., \& Monahan, S. (2018). 'Von anderem Planeten?' Gesture, embodiment, and virtual environments in the orbit of (a)tonality. In N. Reyland \& R. Thumposton (Eds), Music, analysis and the body: experiments, explorations, and embodiments (Vol. 6, pp. 31-50). Leuven, Belgium: Peeters.

Eerola, T., \& Toiviainen, P. (2004). MIDI toolbox: MATLAB tools for music research. Jyväskylä, Finland: University of Jyväskylä.

Kostka, S., \& Payne, D. (2008). Tonal harmony (6th ed.) New York, NY: McGraw-Hill Higher Education.

Krumhansl, C. L. (1990). Cognitive foundations of musical pitch. New York, NY: Oxford University Press.

Lartillot, O., \& Toiviainen, P. (2007). A Matlab toolbox for musical feature extraction from audio. In S. Marchand (Ed.), Proceedings of the 10 International Conference on Digital Audio Effects (DAFx-07) (pp. 237-244). Bordeaux, France: Université Bordeaux

Lartillot, O., Toiviainen, P., \& Eerola, T. (2008). A matlab toolbox for music information retrieval. In C. Preisach, H. Burkhardt, L. Schmidt-Thieme, \& R. Decker (Eds), Data analysis, machine learning and applications (pp. 261-268). Berlin, Germany: Springer. https://doi.org/10.1007/978-3-540-78246-9_31

Poldrack, R. (2020). Statistical thinking for the 21st century: an open source textbook for statistics, with companions for $R$ and Python. Online textbook retrieved from https://statsthinking21.github.io/statsthinking21-core-site/ 